\title{
THE EFFECT OF APPLYING NURSING CARE BUNDLE ON CONTROLLING CENTRAL VENOUS LINE INFECTION IN NEONATAL INTENSIVE CARE UNITS \\ Nadia Mohammed Alfar ${ }^{1}$, Ohoud Youssef EL-Sheikh ${ }^{2}$, Rabab EL sayed Hassan $^{3}$, Mohga Abd Elaziz Selim ${ }^{4}$ \\ ${ }^{1}$ Demonstrator in Pediatric Nursing Department, Faculty of Nursing, Kafr El Sheikh University, Egypt \\ ${ }^{2}$ Lecturer of Pediatric Nursing, Faculty of Nursing, Mansoura University, Egypt \\ ${ }^{3}$ Assist. Prof. Of Pediatric Nursing, Faculty of Nursing, Mansoura University, Egypt ${ }^{4}$ Prof. Of Medical Surgical Nursing, Faculty of Nursing, Kafr El Sheikh University, Egypt E.mail address : doreedode@yahoo.com
}

\begin{abstract}
Central venous line (CVL) is a necessary device used frequently in neonatal intensive care units (NICUs) for critically ill neonates who need continuous monitoring and resuscitation treatment. Significant evidence has accumulated indicating that CVL nursing care bundle is considered as an essential component of CVL infection prevention practice to combat the incidence of CVL infection. Aim: This study aimed to examine the effect of applying nursing care bundle on controlling central venous line infection in neonatal intensive care units. Subject and method: A quasi-experimental design was used. The study was conducted at NICU at Kafr El Sheikh General Hospital. The study included a convenience sample of all available bedside/ working nurses $(n=30), 79$ preterm infant (recruited for 3 months' period). Tools: Three tools were used to collect data. Tool 1: An interview questionnaire for nurse's knowledge. Tool 2: Observational checklist used to assess nurses' performance regarding CVL care bundle. Tool 3: Laboratory investigations tool for preterm infants. Results: More than half of studied nurses $(56.7 \%)$ didn't receive any training program about CVL care bundle for controlling CVL infection in NICU and the majority of the studied nurses had insufficient knowledge, as well as the majority of the studied nurses, had an unsatisfactory practice regarding CVL care bundle preprogram. The sufficient knowledge level and satisfactory practices had improved immediately post and 3 months post program implementation. About half $(51,9 \%)$ had negative CRP post-program implementation, while $(54,4 \%)$ of neonates had negative blood culture post-program. Conclusion: The program had a positive effect on the NICU nurses' knowledge \& practices immediately post\& post 3 months of the program implementation. Recommendation: Including CVL nursing care bundle practices into nurses' routine care and developing regular and continuous educational programs for the nurses in NICU according to their needs aiming to upgrading their knowledge and improving their practice for critically ill neonates.

Keywords: Bundle, Care, Intensive, Nurses, Practice, Neonates, Central Venous Line, Infection.

\section{Introduction}

Central venous lines (CVLs) are considered life lines indispensable in neonatal intensive care units (NICUs). Insertion of an intravascular line is the most common invasive procedure in the

NICU. CVLs are a vital importance to optimize management in NICU. Neonates admitted to NICU in particular preterm and critically ill neonates who are slow to tolerate the introduction of enteral feeding-
\end{abstract}


Nadia Mohammed Alfar et. al.

rely highly on venous access for administering hyperosmolar fluids and medications, parenteral nutrition, and blood products, thus it is crucial to their survival (Ismail, 2015).

The most common types of CVLs used in NICUs are umbilical venous catheters (UVC), peripherally inserted central catheters (PICC), and tunneled central venous line (CVL) e.g., Hickman and Broviac, they are often used in the care of preterm infants. Although CVLs in neonates have many benefits, they have also serious disadvantages. CVL use has been associated with an increased risk for developing central line-associated bloodstream infection (CLABSI), which is a major cause of concern worldwide (Kulali, Çalkavur, Oruç, Demiray \& Devrim, 2019).

Preterm infants are vulnerable age group to infection due to; immunological immaturity, a host of invasive diagnostic and therapeutic procedures, and frequent contact with hospital personnel. CLABSI is the most frequent hospital-acquired infection and is associated with significant morbidity and increase healthcare costs (Chen et al., 2017).

Central line-associated bloodstream infection (CLABSI) was defined, according to the Centre for Disease Prevention and Control (CDC), National Healthcare Safety Network (NHSN), as a primary blood stream infection (BSI) in a patient that had a central line within the 48-hour period before the development of the BSI and there is no evidence of bloodstream related to an infection at another site (CDC, 2017). On the other hand, about $12 \%$ to $25 \%$ of the mortality rate among neonates worldwide attributed to CLABSI, while the healthcare costs vary by each country and healthcare system (Karagiannidou, Zaoutis,
Maniadakis, Papaevangelou \& Kourlaba, 2019).

Approximately 250,000 CLABSIs are acquired each year in US hospitals with death occurring in 28,000 cases. The estimated cost to treat each patient is $\$ 29,156$ and places a $\$ 2.3$ billion burden on the US health care system each year. Additionally, the centers for Medicare and Medicaid services classify CLABSI a never-events preventing hospitals from obtaining reimbursement for treating these infections (Savage et al., 2018).

European CDC held the first Europe-wide point-prevalence survey of health-care-associated infections in pediatric hospitals and reported that the most common type of infection was bloodstream infection 45\%, followed by lower respiratory tract infections $22 \%$, gastrointestinal infections $8 \%$, eye, ear, nose, and throat infections $7 \%$, urinary tract infections 5\% and surgical-site infections $4 \%$. The prevalence of infections was highest in pediatric intensive care units (PICUs) $15.5 \%$, and NICUs 10.7\% (Zingg et al., 2017).

National surveillance of health care-associated infections in Egypt reported that, $30 \%$ were bloodstream infections, $29 \%$ were surgical site infections, $26 \%$ were pneumonia, and $15 \%$ were urinary tract infections (Talaat et al., 2016). Moreover, in Mansoura university children's hospital, pneumonia was the most frequently occurring infection $(11.3 \%)$ followed by bloodstream infection $(8.8 \%)$. The most frequently isolated organisms were Klebsiella $(33.3 \%)$ followed by Escherichia coli 21.6\% (Abdel-Wahab, Ghoneim, Khashaba, El-Gilany \& Abdel-Hady, 2013).

There are a multifactorial risks associated with the development of blood stream infection in neonates, which 
defined as fetal, maternal and NICUs environmental factors. Apart from the immature immune system, some of the fetal factors that predispose neonates to infection are including low birth weight, gestational age and prolonged hospital stay, invasive procedures, endotracheal tubes, parenteral nutrition, lack of adequate hand washing by hospital personnel and indiscriminate use of antibiotics (Dhaneria et al., 2018).

Central line-associated bloodstream infection (CLABSI) represent a significant threat to neonatal safety. Evidenced-based practices, such as CVL care bundle and health care staff education have been shown to decrease CLABSI. A care bundle is a group of evidence-based practices that when performed collectively and consistently implemented together, have been proven to improve the quality of patient care (Kramer, Doellman, Blaser, Rosenberg \& Bernatchez, 2019).

Many CLABSI can be prevented by utilizing proven strategies for CVL insertion and maintenance. Understanding risk factors for CLABSI, educating health care providers regarding prevention practices, ensuring adequate healthcare staffing, and adhering to CVL care bundle are all components of an effective prevention strategies (Schmid, Geffers, Wagenpfeil \& Simon, 2018).

Focused attention on consistent adherence to the use of CVL insertion and maintenance care bundle produced sustained, continually decreasing CVL infection rates. The $\mathrm{CDC}$ and the Asia Pacific Society of Infection Control (APSIC) have recommended evidencebased practices of the CVL care bundles, using a comprehensive educational approach to improve the process of CVL insertion and maintenance and so to reduce the incidence of CLABSI (Kulali,
Çalkavur, Oruç, Demiray \& Devrim, 2019).

Central venous line (CVL) care bundle include; hand hygiene, maximal barrier precautions, skin antisepsis, optimal CVL selection, daily review of line necessity with the removal of unnecessary lines. Nursing adherence with evidence-based practices care bundle is the most important tool in reducing the incidence of CLABSI and improving premature infant's outcome (Payne, Hall, Prieto \& Johnson, 2017).

The aim of this study was to:

Examine the effect of applying nursing care bundle on controlling central venous line infection in neonatal intensive care units.

\section{Research hypothesis}

- Applying nursing care bundle will improve nurses' knowledge about controlling central venous line infection in neonatal intensive care units.

- Applying nursing care bundle will improve nurses' practice of controlling central venous line infection in neonatal intensive care units.

- Applying nursing care bundle will reduce the incidence of central venous line infection among preterm infants in neonatal intensive care units.

\section{Subjects and Method:}

Research Design:

A quasi-experimental design was utilized in carrying out the study.

\section{Setting:}

The study was conducted in at NICU at Kafr El Sheikh General Hospital. Subjects:

- A convenience sample of all available bedside/ working nurses $(\mathrm{N}=30)$, who were available during the period of data collection in the previously mentioned setting and willing to participate in this study, regardless 
their ages, educational level or years of experience.

- All premature infants who were admitted to the previously mentioned setting through the period of 3 months.

\section{Inclusion criteria}

- Both gender.

- Indicated for CVL insertion

- Had CVL

\section{Exclusion criteria}

- Premature infants who had a bloodstream infection.

Tools of Data Collection:

Data were collected through the following tools:

Tool I: A structured interview questionnaire for nurses (pre, post immediate \& follow up):

It was designed by the researcher in a simple Arabic language after reviewing the related literature. It composed of multiple-choice questions that were collected by the researcher through an interview with nurses to assess their knowledge about all aspects of CVL nursing care bundle in NICUs. It comprised of two parts as follows:

Part 1:

Concerned with socio-demographic characteristics of the studied nurses such as age, level of education, years in the profession, years of experience in NICUs, and previous attendance of training program related to nursing care bundle for controlling CVL infection.

Part 2:

Concerned with nurses' knowledge about CVL and CVL infection (pre, post immediate and follow up). It was composed of 32 questions about; definition of CVL, common sites of CVL insertion, forbidden sites of CVL insertion, the causes of CVL insertion, definition of CLABSI, risk factors for CVL infection, signs \& symptoms of CVL infection, and complications of CVL infection. Furthermore, this part assess the nurses' knowledge about bundle of care for controlling CVL infection (pre, post immediate, follow up), which covered the following items: definition of nursing care bundle, items of nursing care bundle, barrier precautions, elements of the daily nursing care bundle, disinfect substance, recommended time to change dressing on the CVL site, and the barriers facing nurses to adhere with bundle of care for controlling CVL infection in practice.

\section{Scoring system}

According to the answers were collected from the nurses related to their knowledge; the scoring system was distributed as follows: the correct complete answer was given two grades, while the correct incomplete answer was given one grade and zero was given for incorrect, missed or unknown answer according to (Taha, 2014). The total score of the studied nurse's knowledge was 66 grades.

Accordingly, those who obtained $80 \%$ or more were classified as adequate level of knowledge, and below 80\% inadequate level of knowledge.

Tool II: Nurses' Observation checklist (pre, post immediate \& follow up).

It was developed by the Centers for Disease Control and Prevention (CDC, 2017) and was adapted to suit the policies and procedures at NICUs. Data was collected by the researcher to assess nurses' practices as regarding bundle of care to control CVL infection in NICUs pre, post immediate and 3 months following the implementation of the program. The audit checklist contains two parts:

Part (1): Infection Control Practices Observational Checklist.

It was adopted from the Centers for Disease Control and Prevention 
(CDC, 2017). It was used to observe nurses' practices directly through audit checklist about hygienic hand washing, gloving and gowning following aseptic technique.

\section{Scoring system}

Two options for every step done correctly(1mark) and done incorrect / incomplete / not done (no marks). The total score of the studied nurses' practices was estimated according to median score into a satisfactory level of performance $80 \%$ or more, and unsatisfactory level of performance less than $80 \%$.

Part (2): Nursing Care Of Central Venous Line Observational Checklist:

It was adapted from the Centers for

Disease Control and Prevention (CDC, 2017) to collect the required data through direct observation of the nurses in the NICUs, using predesigned checklists to assess the actual nurses' practice of care during insertion and daily care.

\section{Scoring system:}

Two options for every step done correctly(1mark) and done incorrect / incomplete / not done (no marks). The total score of the studied nurses' practices was estimated according to median score into a satisfactory level of performance $80 \%$ or more, and unsatisfactory level of performance less than $80 \%$.

Tool (III): Laboratory investigations tool.

This tool was the clinical indicators of neonates to measure and report the blood stream infection before and after implementation for the bundle of care of CVL. It was taken immediately after 48 hours of the insertion. Centers for Disease Control and Prevention (CDC, 2016). It was composed of two main laboratory investigations, which are; Creactive protein (CRP) of the studied premature infants and premature infants blood culture.

\section{Operational design.} Preparatory phase:

This phase included a review of the past and current related literature and studies, using available books, periodicals, magazines, and articles to be acquainted with the various aspects of the study research problem and develop the study tool. The guiding booklet was prepared by the researcher. It was specially designed in a simple Arabic language to meet nurses' practical needs and knowledge deficits regarding nursing care bundle for controlling CVL infection in NICU. The content validity of the study tools was assessed and revised by a panel of five experts in the field of nursing care to participate in the evaluation and revision of the tools. Rewording of some items was recommended to improve the clarity content, sequence of items and relevance or irrelevance of content.

According to their suggestions, the required modifications were done and the internal consistency of the developed tools was tested by using Cronbach's alpha coefficient test.

\section{Pilot study:}

A pilot study was carried out on $10 \%$ of the total subjects' size 3 nurses and 8 of premature infants in NICU according to the criteria of selection before starting the data collection to test the tools clarity, feasibility, and applicability. As well to estimate the time needed to apply the study tools and to detect any modification. The tools did not require any modification. Therefore, the participated subjects of the pilot study were not included in the study sample.

Field work

Data collection period:

- Data collection extended over a period of six months from the first of January 2019 to the end of June 2019. 
- The researcher started by explaining the aim and nature of the study to the studied nurses.

- The framework of the study was carried out to 4 phases as the following:

i) Assessment phase

- Each nurse was interviewed individually before applying the educational nursing care bundle in order to collect the baseline data using the study tool I part (I)

- Assessment of nurses' knowledge about nursing care bundle for controlling CVL infection in NICU was performed using a tool I part (2)

- Assessment of nurses' practices about nursing care bundle for controlling CVL infection in NICU was performed using a tool (II) part $(1,2)$

- Assess the outcome of applying nursing care bundle through the neonatal clinical indicators (CRP\& blood culture) of the studied premature infants by using a tool (III)

\section{ii) Planning phase}

- Based on the findings of the assessment phase goals, priorities and expected outcomes were formulated to meet nurses' practical needs and knowledge deficits regarding nursing care bundle, CVL, CVL infection in NICU and its control.

- In this phase, four sessions were planned by the researcher for the nurses to provide them with practical knowledge about CVL, CVL infection in NICU and all aspects of nursing care bundle.

iii) Implementation phase

- The researcher was available four days/ week from Sunday to Wednesday at morning, afternoon, and night shifts. The studied nurses divided into subgroups according to their numbers of each shift (6-10).
Morning shifts sessions started from 7 am to $9 \mathrm{am}$, afternoon shifts from $1 \mathrm{pm}$ to $3 \mathrm{pm}$, and night shifts from $8 \mathrm{pm}$ to $10 \mathrm{pm}$.

- The studied nurses were divided into five groups; each one consisted of 6 to 10 nurse.

- Both the first and second sessions were theoretical parts conducted in the classroom placed in NICU. In the first session, the researcher introduce herself, welcomed the nurses, explain the objectives of the sessions, then gathering the demographic characteristics.

- The third and four sessions were practical part conducted to observes nurses' practices pre and post implementation of the nursing care bundle

- Guiding colored booklet about CVL infection and all aspects of nursing care bundle was given to each nurse after the assessment phase (during the first session) for attracting her attention, motivated her and help her for reviewing its content when needed.

- During the session, the researcher used questions, discussion and different teaching methods as group discussion, question and electronic brainstorming such as (video conference), demonstration and redemonstration. Numerous didactic aids were used, such as handout guideline and power point.

- Brief, clear and simple words used during the session by the researcher. As well as at the end of each session, a brief summary was given.

- The Assessment of preterm infant were started one month before implementation of the program.

- The laboratory results for CRP and Blood culture were collected for all 
preterm infant in the NICU after two days of CVL insertion pre-program implementation during the period of 8 weeks.

- The total number of preterm infant were 84 . Five of them were excluded, as their clinical indicators for CVL infection were positive. Seventy-nine of neonates assessed for CVL infection post the program implementation before neonatal discharge with 24 hours, during the period of 16 weeks. From the total number of preterm infant (79), nine were dead all over the period of data collection.

\section{iv) Evaluation phase}

- Every nurse was interviewed separately after application of the nursing care bundle in order to evaluate the level of knowledge and practices by using tool (I) part $2 \&$ tool (II) part $(1,2)$ during Immediate post-test and follow up (after 3 months).

\section{Administrative design:}

Official approval was obtained from the Research Ethical Committee of Mansoura Faculty of nursing to conduct the study.

- An official letter to conduct the study was obtained from the hospital administrative authority. In the previously mentioned setting which affiliated to the neonatal intensive care unit in Kafr El Sheikh General Hospital after explaining the purpose of the study

- An official letter to conduct the study was obtained from the heads of the neonatal intensive care unit. in the previously mentioned setting which affiliated to the neonatal intensive care unit in Kafr El Sheikh General Hospital after explaining the purpose of the study
Ethical considerations:

The researcher followed ethical research principles as the following:

- Oral approval was obtained from each participant (nurses) before the start of the study after the explanation of the purpose of the study.

- An oral approval was obtained from any of preterm infants' parents (father or mother) before the beginning of the study after explanation of the study purpose.

- The researcher emphasized that the study cause no physiological or psychological harm to the neonate patients.

- Privacy and confidentiality of the collected data were assured throughout the whole study phases.

- Any participant has the right to withdraw from the study at any time without any responsibility.

\section{Statistical design:}

The collected data was coded and inserted into a data file using the Excel program for Windows. Frequency analysis and manual review were used to detect errors. After full insertion, the data were transformed into the version of the Statistical Social Science Package (SPSS) by which the analysis was conducted applying frequency tables with percentage. Data were revised, coded and analyzed. Qualitative data were presented as number and percent. The Chi-square test was used for comparison between groups as appropriate, and for comparing between two groups \& Paired t test used for comparison between two paired groups, while Pearson correlation was used for correlation between continuous parametric data. All test were performed at a level of significance $(\mathrm{P}$ - value) equal or less than was considered to be statistically significant. Quantative data were described as mean \pm SD (standard 
deviation) for parametric and Median data for non-parametric data. Anova test was used for comparing between more than two groups ( pre-post,follow program), and association between personal characteristics. Finally, linear regression was used for prediction of the relation between independent variables and chosen dependent variables. They were testes for normality by Kolmogorov-Smirnov test.

\section{Level of significance:}

The level of significance appeared when the probable error

- Significant when the probability of error is less than $5 \%(\mathrm{p}<0.05)$.

- Highly significant when the probability of error is less than $0.1 \%$ $(\mathrm{p}<0.001)$.

\section{Results:}

Distribution of the studied nurses according to their demographic characteristics are proved in table (1), this table revealed that, $(43.3 \%)$ of the studied nurses were in the age group from 20 to less than 30 years, and more than one third of them $(36.7 \%)$ were graduated from technical institute. As regards to years of experience more than three quarters of nurses $(76.7 \%)$ had less than 5 years working in the NICU, while only (16.7\%) of them had 5 to less than 10 years of experience. In addition, more than half of the NICU nurses $(56.7 \%)$ did not attend previous training program about CVL nursing care bundle.

Table (4): clarified mean scores of nurses' knowledge about CVL,CVL infection and nursing care bundle to control CVL infection in NICU pre, immediately post and post 3 months of the program implementation. This table indicated that, the mean scores of nurses' knowledge about CVL, CVL infection and nursing care bundle in NICU was 28.5 \pm 5.9 . This mean improved to $45.16 \pm 5.4$ and $41.6 \pm 3.32$ immediately post and post 3 months of the program implementation respectively, with statistically significant differences at $\mathrm{p}<$ 0.001 .

Table (5): clarified mean scores of nurses' practices regarding nursing care bundle to control CVL infection in NICU pre, immediately post and post 3 months of the program implementation. This table illustrated that, the mean scores of nurses' practices regarding nursing care bundle to control CVL infection in NICU was $33.7 \pm 6.25$. This mean improved to $54.33 \pm 4.23$ and $51 \pm 4.53$ immediately post and post 3 months of the program implementation respectively, with statistically significant differences at $\mathrm{p}<$ 0.001 .

Table (6): clarified a relation between total nurses' knowledge and practices pre, immediately post and post 3 months of program implementation. As regards association between nurses' knowledge and infection control practices pre, immediately post and post 3 months of the program implementation. It was showed that, there was a significant positive association between nurses' knowledge and infection control practices immediately post and post 3 months of the program implementation with a highly statistical significant differences at $(\mathrm{p}=$ $0.011 \& p=0.021)$ respectively .

The distribution of NICU patients according to their general characteristics is illustrated in (Table (9); It was found that, $43 \%$ of the studied neonates were in the age group from 27- 29 weeks of age. In relation to gender, $47 \%$ were males. Regarding neonates' birth weight, it was found that $(43 \%)$ were very low birth weight less than 1500 G. Furthermore, $72.2 \%$ of the studied neonates had a diagnosis of respiratory system diseases.

Table (10): clarified neonatal clinical indicators of CVL infection during 
THE EFFECT OF APPLYING NURSING CARE etc...

pre, post implementation of the program. It was reported that, less than two-thirds (63.3\%) of neonates had positive CRP preprogram implementation, while about half of them $(51,9 \%)$ had negative CRP postprogram implementation. Furthermore, about half of them $(54,4 \%)$ had positive blood culture pre-program implementation, while $(55,6 \%)$ of neonates had negative blood culture postprogram implementation.

Table (10): presented correlation between infection control practices and neonatal clinical indicators of CVL infection pre, immediately post and post 3 months of program implementation. This table demonstrated that, there was a significant positive relation between infection control practices and CRP preprogram implementation $(\mathrm{r}=7.053, \mathrm{p}=$ 0.039). As well as, there was a significant positive relation between infection control practices and both clinical indicators (CRP, Blood culture) immediately after and post 3 months of program implementation $(r=17.9, p=0.001) \&(r$ $=11.01, \mathrm{p}=0.001)$ respectively

Table (13): clarified a relation between general characteristics of the neonates and neonatal clinical indicators of CVL infection pre and post-program implementation. This table illustrated the relation between characteristics of the neonates and their clinical indicators of CVL infection pre and post the program implementation. It indicated that, there was statistically significant relation between CRP and birth weight post program implementation $(\mathrm{P}=0.023)$ as well blood culture and their age in weeks $(\mathrm{P}=$ 0.014).

Table (1): Distribution of the studied nurses according to their demographic characteristics $(\mathrm{N}=30)$.

\begin{tabular}{|c|c|c|}
\hline Characteristics & Nurses $(n=30)$ & $\%$ \\
\hline \multicolumn{3}{|l|}{ Age in years } \\
\hline$<\mathbf{2 0}$ & 10 & 33.3 \\
\hline $20:<30$ & 13 & 43.3 \\
\hline $30: 40$ & 6 & 20 \\
\hline$>40$ & 1 & 3.3 \\
\hline \multicolumn{3}{|l|}{ Educational level } \\
\hline Diploma degree & 8 & 26.7 \\
\hline Technical institute & 11 & 36.7 \\
\hline Bachelor degree & 7 & 23.3 \\
\hline Post graduate & 4 & 13.3 \\
\hline \multicolumn{3}{|l|}{ Years of nursing experience } \\
\hline$>5$ years & 22 & 73.4. \\
\hline $5 \leq 10$ years & 5 & 16.7 \\
\hline$>10$ years & 3 & 10 \\
\hline \multicolumn{3}{|l|}{ Years of experience in NICU } \\
\hline$>5$ years & 23 & 76.7 \\
\hline $5 \leq 10$ years & 5 & 16.7 \\
\hline$>10$ years & 2 & 6.7 \\
\hline \multicolumn{3}{|c|}{ Attending previous training about CVL nursing care bundle } \\
\hline No & 17 & 56.7 \\
\hline Yes & 13 & 43.3 \\
\hline
\end{tabular}


Nadia Mohammed Alfar et. al.

Table (4); Mean scores of nurses' knowledge about CVL,CVL infection and nursing care bundle to control CVL infection in NICU pre, immediately post and post 3 months of the program implementation $(\mathrm{N}: 30)$.

\begin{tabular}{|l||c|c|c|c|c|}
\hline \multicolumn{1}{|c|}{ Knowledge items } & \multicolumn{3}{|c|}{ Programs phases } & \multirow{2}{*}{$\begin{array}{c}\text { F- } \\
\text { value }\end{array}$} & P \\
\cline { 2 - 5 } & Pre & $\begin{array}{c}\text { Immediately } \\
\text { post }\end{array}$ & $\begin{array}{c}\text { Post 3 } \\
\text { months }\end{array}$ & & \\
\cline { 2 - 5 } & Mean \pm SD & Mean \pm SD & Mean \pm SD & & \\
\cline { 1 - 5 } $\begin{array}{l}\text { Nurses' knowledge about } \\
\text { CVL. }\end{array}$ & $4.16 \pm 1.9$ & $7.53 \pm 0.97$ & $5.86 \pm 1.69$ & 33.45 & $\mathbf{0 . 0 0 1 *}$ \\
\hline $\begin{array}{l}\text { Nurses' knowledge about } \\
\text { CVL infection that } \\
\text { neonate may be exposed } \\
\text { to in NICUs. }\end{array}$ & $7.23 \pm 2.5$ & $11 \pm 1.68$ & $10.39 \pm 1.12$ & 35.14 & \multirow{0}{*0.001*}{} \\
\hline $\begin{array}{l}\text { Nurses' knowledge about } \\
\text { nursing care bundle to } \\
\text { control CVL infection in } \\
\text { NICU. }\end{array}$ & $17.1 \pm 3.86$ & $26.63 \pm 4.14$ & $25.46 \pm 2.43$ & 63.29 & $\mathbf{0 . 0 0 1 *}$ \\
\hline Total nursing knowledge & $28.5 \pm 5.9$ & $45.16 \pm 5.4$ & $41.6 \pm 3.32$ & 90.94 & $\mathbf{0 . 0 0 1 *}$ \\
\hline
\end{tabular}

Table (5); Mean scores of nurses' practices regarding nursing care bundle to control CVL infection in NICU pre, immediately post and post 3 months of the program implementation $(\mathrm{N}: 30)$.

\begin{tabular}{||c|c|c|c|c|c|}
\hline \multirow{2}{*}{ Nurses' practices } & \multicolumn{3}{|c|}{ Programs phases } & \multirow{2}{*}{$\begin{array}{c}\text { F- } \\
\text { value }\end{array}$} & P \\
\cline { 2 - 6 } & Pre & $\begin{array}{c}\text { Immediately } \\
\text { post }\end{array}$ & $\begin{array}{c}\text { Post 3 } \\
\text { months }\end{array}$ & & \\
\cline { 2 - 6 } Mean \pm SD & Mean \pm SD & Mean \pm SD & & \\
\hline $\begin{array}{l}\text { Infection control } \\
\text { practices. }\end{array}$ & $14.1 \pm 5.67$ & $23.36 \pm 3.04$ & $20.96 \pm 4.07$ & 35.878 & $\mathbf{0 . 0 0 1 *}$ \\
\hline $\begin{array}{l}\text { Central venous line } \\
\text { care during insertion. }\end{array}$ & $10 \pm 1.11$ & $14.33 \pm 0.95$ & $13.46 \pm 1.4$ & 34.563 & $\mathbf{0 . 0 0 1 *}$ \\
\hline $\begin{array}{l}\text { Central venous line } \\
\text { daily care. }\end{array}$ & $9.6 \pm 1.03$ & $16.63 \pm 2.01$ & $16.56 \pm 1.75$ & 79.48 & $\mathbf{0 . 0 0 1 *}$ \\
\hline $\begin{array}{l}\text { Total practice for } \\
\text { controlling } \\
\text { infection }\end{array}$ & $33.7 \pm 6.25$ & $54.33 \pm 4.23$ & $51 \pm 4.53$ & 42.132 & $\mathbf{0 . 0 0 1 *}$ \\
\hline
\end{tabular}


THE EFFECT OF APPLYING NURSING CARE etc...

Table (6); Relation between total nurses' knowledge and practices pre, immediately post and post 3 months of program implementation $(\mathrm{N}: 30)$.

\begin{tabular}{|c|c|c|c|c|}
\hline \multirow{2}{*}{\multicolumn{2}{|c|}{ Variables }} & \multicolumn{3}{|c|}{ Nurses' knowledge } \\
\hline & & \multirow{2}{*}{$\begin{array}{c}\text { Pre } \\
\mathrm{r}=0.23 \\
\mathrm{p}=0.905\end{array}$} & \multirow{2}{*}{$\begin{array}{c}\text { Immediately } \\
\text { post }\end{array}$} & \multirow{2}{*}{ Post 3 months } \\
\hline \multirow{3}{*}{$\begin{array}{c}\text { Infection } \\
\text { control } \\
\text { practices }\end{array}$} & Pre & & & \\
\hline & Immediately post & & $\begin{array}{c}\mathrm{r}=7.021 \\
\mathrm{p}=0.011 *\end{array}$ & \\
\hline & Post 3 months & & & $\begin{array}{c}r=5.191 \\
p=0.021 *\end{array}$ \\
\hline
\end{tabular}

Table (9); Distribution of NICU patients according to their general characteristics (N=79)

\begin{tabular}{|c|c|c|}
\hline Variables & No & $\%$ \\
\hline \multicolumn{3}{|l|}{ Age in weeks } \\
\hline $27-29$ & 34 & 43 \\
\hline $30-32$ & 23 & 29.2 \\
\hline $33-35$ & 22 & 27.8 \\
\hline Range (27-35) & \multicolumn{2}{|c|}{ Mean \pm SD $30.39 \pm 2.5$} \\
\hline \multicolumn{3}{|l|}{ Birth Weight } \\
\hline Low Birth Weight Less Than $2500 \mathrm{G}$ & 32 & 40.5 \\
\hline Very Low Birth Weight Less Than $1500 \mathrm{G}$ & 34 & 43 \\
\hline Extremely Low Birth Weight Less Than $1000 \mathrm{G}$ & 13 & 16.5 \\
\hline \multicolumn{3}{|l|}{ Mean \pm SD $1850 \pm 115$} \\
\hline \multicolumn{3}{|l|}{$\overline{\text { Sex }}$} \\
\hline Male & 47 & 59.5 \\
\hline Female & 32 & 40.5 \\
\hline \multicolumn{3}{|l|}{ Medical diagnosis } \\
\hline Respiratory system diseases & 57 & 72.2 \\
\hline Cardiovascular diseases & 22 & 27.8 \\
\hline
\end{tabular}


Nadia Mohammed Alfar et. al.

Table (10); Neonatal clinical indicators of CVL infection during pre, post implementation of the program.

\begin{tabular}{|c|c|c|c|c|c|c|c|}
\hline \multirow{2}{*}{ variables } & \multirow{2}{*}{ Category } & \multicolumn{2}{|c|}{ Pre $(n=79)$} & \multicolumn{2}{|c|}{$\operatorname{Post}(n=70)$} & \multirow{2}{*}{$\mathbf{X}^{2}$} & \multirow{2}{*}{$\mathbf{P}$} \\
\hline & & No & $\%$ & No & $\%$ & & \\
\hline \multirow[t]{2}{*}{ CRP } & Positive & 50 & 63.3 & 30 & 48.1 & \multirow{2}{*}{0.968} & \multirow{2}{*}{0.227} \\
\hline & Negative & 29 & 36.7 & 40 & 51.9 & & \\
\hline \multirow{2}{*}{$\begin{array}{c}\text { Blood } \\
\text { culture }\end{array}$} & Positive & 43 & 54.4 & 26 & 44.4 & \multirow{2}{*}{0.602} & \multirow{2}{*}{0.374} \\
\hline & Negative & 36 & 45.6 & 44 & 55.6 & & \\
\hline
\end{tabular}

*Significant $(\mathbf{P}<\mathbf{0 . 0 5})$.

Table (13); relation between general characteristics of the neonates and neonatal clinical indicators of CVL infection pre and post-program implementation $(n=79)$.

\begin{tabular}{|c|c|c|c|c|c|c|c|}
\hline \multirow{2}{*}{\multicolumn{2}{|c|}{ Items }} & \multirow{2}{*}{\multicolumn{3}{|c|}{ Age "in weeks" }} & \multirow{2}{*}{\multicolumn{3}{|c|}{ Birth Weight }} \\
\hline & & & & & & & \\
\hline \multirow{2}{*}{$\begin{array}{l}\text { CRP } \\
\text { Pre } \\
\text { program }\end{array}$} & Positive & 23 & 13 & 14 & 21 & 23 & 6 \\
\hline & Negative & 11 & 10 & 8 & 11 & 11 & 7 \\
\hline \multicolumn{2}{|l|}{ Sig $\left(X^{2}\right)$} & \multicolumn{3}{|c|}{0.694} & \multicolumn{3}{|c|}{0.335} \\
\hline \multirow{2}{*}{$\begin{array}{l}\text { CRP } \\
\text { post } \\
\text { program }\end{array}$} & Positive & 13 & 9 & 8 & 16 & 10 & 4 \\
\hline & Negative & 18 & 11 & 11 & 11 & 20 & 9 \\
\hline \multicolumn{2}{|l|}{$\operatorname{Sig}\left(X^{2}\right)$} & \multicolumn{3}{|c|}{0.964} & \multicolumn{3}{|c|}{$\mathbf{0 . 0 2 3}$ * } \\
\hline \multirow{2}{*}{$\begin{array}{l}\text { Blood } \\
\text { culture } \\
\text { Pre }\end{array}$} & Positive & 19 & 12 & 12 & 18 & 18 & 7 \\
\hline & Negative & 15 & 11 & 10 & 14 & 16 & 6 \\
\hline \multicolumn{2}{|l|}{$\operatorname{Sig}\left(X^{2}\right)$} & \multicolumn{3}{|c|}{0.899} & \multicolumn{3}{|c|}{0.836} \\
\hline \multirow{2}{*}{$\begin{array}{l}\text { Blood } \\
\text { culture } \\
\text { Post }\end{array}$} & Positive & 11 & 6 & 9 & 9 & 10 & 8 \\
\hline & Negative & 20 & 16 & 8 & 19 & 19 & 6 \\
\hline \multicolumn{2}{|l|}{$\operatorname{Sig}\left(X^{2}\right)$} & \multicolumn{3}{|c|}{ 0.014* } & \multicolumn{3}{|c|}{0.227} \\
\hline
\end{tabular}

*Significant $(\mathbf{P}<0.05)$

\section{Discussion}

The current study findings revealed that, nearly two-thirds of the studied nurses were in the age group from 20 to less than 30 years old (Table 1). This result was in accordance with
Ismail \& Mostafa, (2016) who conducted a study about "knowledge and practice of standard precautions among neonatal intensive care unit healthcare workers of Misurata teaching hospital, Libya" and 
stated that, the vast majority of nurses were within the age of 20 to 30 years old.

In relation to the educational level of the studied nurses, the present study showed that, the majority of the studied nurses had technical institute degree, (table 1). This result was incongruence with Mohmmed \&Yousif, (2017) who conducted a study about "effect of health educational program on nurses knowledge \& practice regarding infection control in neonatal intensive care unit at pediatric hospitals in Khartoum State, Sudan" and reported that, the majority of the studied nurses had a diploma certificate. While, Batran, Ayed, Salameh, Ayoub \& Fasfous, (2018) who conducted a study about " Are standard precautions for hospitalacquired infection among nurses in public sector satisfactory?" stated that, most of the studies sample had bachelor degree in nursing.

Regarding nurses' years of experience, the current study clarified that, about two-thirds of the studied nurses had less than 5 years of working experience in the NICU (Table 1). This result was in disagreement with Geberemariyam, Donka \& Wordofa, (2018) who conducted a study about "assessment of knowledge and practices of healthcare workers towards infection prevention and associated factors in healthcare facilities of West Arsi District, Southeast Ethiopia" and found that, the majority of the studied nurses had less than five years of working experience in the NICU.

It is obvious from the current study that, more than half of the studied NICU nurses $(56.7 \%)$ did not receive training a program about CVL infection \& nursing care bundle (Table 1). This finding was in incongruence with Manzo et al., (2019) who conducted a study about "knowledge and behavior of professionals about bundled strategies of central venous catheter" who reported that, the vast majority of nurses had received training program about CVL infection \& nursing care bundle. The finding of the present study might be due to the workload on nurses and shortage of staff may keep them had no more time to attend any training program.

In relation to nurses' knowledge about CVL, the present study revealed that, the total mean knowledge scores of the studied nurses was $28.5 \pm 5.9$ regarding CVL, CVL infection, nursing care bundle preprogram implementation, while the mean knowledge scores increased immediately after the program to $45.16 \pm 5.4$ and post three months of the program implementation decrease to $41.6 \pm 3.32$ (Table 4). This result was in the same line with Cooper, (2019) who conducted a study about "improving nurses' knowledge of central line-associated bloodstream infection" and found that, the mean knowledge scores of nurses has increased from 72.1to 94.1 post-program implementation and decrease to 90.6 in follow up phase. Also, the finding of the current study is in accordance with Pareek, Kausik, Jangir, \& Pareek, (2018) who conducted a study about "effectiveness of planned teaching program regarding knowledge on selected venous access device care among B. Sc nursing final year student" who reported that, there was a significant improvement of nurses' knowledge post the program implementation. The researcher attributed that; the information can be easily forgotten if they are not refreshed periodically. Besides that, nurses may value practices more than knowledge.

It was indicated from the current study that, the majority of the studied nurses have got unsatisfactory level regarding total CVL care practices for controlling CVL infection pre-program implementation 
which improved immediately post and post three months of the program implementation (table 5). The possible explanation for this finding may due to that more than half of the studied nurses did not receive training program about CVL infection \& nursing care bundle. This result was supported by Dumpa et al., (2016) who conducted a study about "reduction in central line-associated bloodstream infection rates after implementations of infection control measures at a level 3 neonatal intensive care unit" who reported that, a few numbers of nurses used infection control practices to control CVL infection before they received the educational program. From the researcher's point of view, this result might be related to other factors such as shortage of nurses, unavailability of hospital protocols and polices for caring CVL, and unavailability of workshops and educational programs.

Concerning association between nurses' knowledge and infection control practices pre, immediately after and post 3 months of the program implementation (table 6). The result of the same study proved that, there was a significant positive association between nurses' knowledge and their infection control practices immediately after and post 3 months of the program implementation with a highly statistical significant differences at $p<0.001$. While there is no significant correlation pre the program implementation. This result was in contradiction with Deshmukh \& Shinde, (2014) who emphasized that, there was a significant negative correlation between nurses' knowledge and practice regarding CVL prevention before and after the training program. From the researcher's point of view, these current findings may relay to the nurses' knowledge act as an important and independent factor in carrying out infection control practices.

Concerning the characteristics of the studied neonates, the current study reflected that, more than two-thirds of the studied neonates were low birth weight, and the majority of them ranged (27-29) weeks (table 9). these findings were uncoordinated with Freitas, Araujo, Melo \& Romero, (2019) who conducted a study about "lateonset sepsis and mortality among neonates in a Brazilian intensive care unit " and stated that, the majority of the studied neonates were very low birth weight.

As well, the current study showed that, more than half of the studied neonates were males (table 9). This finding is identical to the finding of Smulders, van Gestel \& Bos, (2013) who conducted a study about "Are central line bundles and ventilator bundles effective in critically ill neonates and children?" and mentioned that, males represent the higher percentages than the girls at NICU. While, this result disagreed with Bannatyne, Smith, Panda, Abdel-Latif \& Chaudhari, (2018) who stated that, the majority of neonates were females. Moreover, the present study indicated that, the majority of neonates diagnosed with respiratory diseases. (table 9), which is not similar to McMullan \& Gordon, (2016) who found that, the most common diagnosis among pediatric NICU patients in Sydney, Australia was a cardiac diseases.

Regarding the clinical indicators of CVL infection among neonates, the present study illustrated that, about half of the neonates $(51,9 \%)$ had negative CRP postprogram implementation. Furthermore, about half $(55,6 \%)$ of neonates had negative blood culture post-program implementation (table 10). This finding was in the same line with Schmid, Geffers, Wagenpfeil \& Simon, (2018) who conducted a study about "preventive 
bundles to reduce catheter-associated bloodstream infections in neonatal intensive care" and revealed that, there was statistically significant reduction in CVL infection rate following the introduction of care bundle. From the researcher point of view, the improvement in the clinical indicators of CVL infection among neonates not only related to the implementation of CVL care bundle but also to many variables in NICU as, antibiotics use.

As regards the relation between general characteristics of the studied neonates and neonatal clinical indicators of CVL infection (table 13). The current study demonstrated that, there was a positive relation between CRP and birth weight post-program implementation $(\mathrm{P}=0.023)$, this finding was in accordance with Savage, (2018) who stated that, The incidence of CVL infection increases with low birth weight (61.7\% for $<1000 \mathrm{gm}$ and $43.1 \%$ for $<1500$ gm.). The researcher suggested that, the present study result might be related to the risk of $\mathrm{CVL}$ infection increases with a reduction in birth weight, which was found the most important factor in the occurrence of CVL infection.

The finding of the same study found that, there was a significant positive relation between age in weeks and blood culture post-program implementation $(\mathrm{P}=0.014)$ (table 15). This finding was in the same line with Bannatyne, Smith, Panda, Abdel-Latif \& Chaudhari, (2018) who conducted a study about "retrospective cohort analysis of central line associated blood stream infection following introduction of a central line bundle in a neonatal intensive care unit" and mentioned that, the incidence rate of CVL infection increases with reduction in gestational age. The researcher attributed this result to the fact that, most of the NICU patients are premature, they have a mature immune system, which the most important risk factor for CVL infection.

\section{Conclusion}

Based on the finding of the current study, it is concluded that, there was an improvements in the NICU nurses' knowledge and practices after implementation of the CVL nursing care bundle either immediately post or post three months of the program implementation than before its implementation. Additionally, there was a positive significant statistical correlation between total nurses' knowledge scores and attending previous training program immediately post the program implementation. There was a significant positive association between nurses' knowledge and infection control practices immediately post and post 3 months of the program implementation respectively. Finally, About half $(51,9 \%)$ had negative CRP post-program implementation, while $(54,4 \%)$ of neonates had negative blood culture post-program.

\section{Recommendations}

In the light of the findings of the current study, the following recommendations are suggested:

For the nurses

- Providing up-to-dated, regular educational programs about CVL, CVL infection and CVL nursing care bundle should be developed for the health care team members in NICU.

- Information relates to CVL infection and CVL care bundle should be provided in formats and ways as manual booklets and pamphlets that are suited to nurses' level of understanding.

- All sectors of the health care system should be educated/ trained on CVL nursing care bundle to recognize how to 
control CVL infection in NICU and how to initiate and maintain correct and suitable preventive measures.

- Periodically monitoring of the knowledge and adherence to CVL care bundle for all personnel involved in the insertion and maintenance of CVL.

- Use maximal sterile barrier precautions, including the use of a cap, mask, sterile gown, sterile gloves, and a sterile full body drape, for CVL care.

For the hospital' management team:

- Hospital managers should facilitate the accessibility of modern equipment used in the prevention of CVL infection to obtain the quality output of CVL care.

- Hospital managers should communicate the ministry of health to arrange the campaigns for critical care nurses to provide them with adequate information and skills regarding management and prevention of CVL infection which leads to good management practice

For further study:

- Studies to the risk factors and barriers that affect nurses' application to CVL nursing care bundle for the preterm infant.

- Replication of this study with a larger sample at different neonatal intensive care units and with longitudinal follow-up for neonatal measures.

\section{References}

Ismail, M. (2015). STUDY OF DIFFERENT NEONATAL VASCULAR ACCESSES IN NEONATAL INTENSIVE CARE UNITS IN DIFFERENT UNIVERSITIES HOSPITALS IN
EGYPT. AL-AZHAR

ASSIUT

MEDICAL JOURNAL, 13(2), 2.

Kulali, F., Çalkavur, Ş., Oruç, Y., Demiray, N., \& Devrim, İ. (2019). Impact of central line bundle for prevention of umbilical catheter-related bloodstream infections in a neonatal intensive care unit: A pre-post intervention study. American journal of infection control, 47(4), 387-390.

Karagiannidou, S., Zaoutis, T., Maniadakis, N., Papaevangelou, V., \& Kourlaba, G. (2019). Attributable length of stay and cost for pediatric and neonatal central line-associated bloodstream infections in Greece. Journal of infection and public health, 12(3), 372-379.

Chen, Y. C., Lin, C. F., Rehn, Y. J. F., Chen, J. C., Chen, P. Y., Chen, C. H., ... \& Huang, F. L. (2017). Reduced nosocomial infection rate in a neonatal intensive care unit during a 4-year surveillance period. Journal of the Chinese Medical Association, 80(7), 427-431.

Centers for Disease Control and Prevention (2017). Bloodstream infection event (central line-associated bloodstream infection and non-central line-associated bloodstream infection). Atlanta, GA: Centers for Disease Control and Prevention, 4, 132.

Savage, T., Hodge, D. E., Pickard, K., Myers, P., Powell, K., \& Cayce, J. M. (2018). Sustained reduction and prevention of neonatal and pediatric central line-associated bloodstream infection following a nurse-driven quality improvement initiative in a pediatric facility. Journal of the Association for Vascular Access, 23(1), 30-41. 
Zingg, W., Hopkins, S., Gayet-Ageron, A., Holmes, A., Sharland, M., Suetens, C., ... \& Cairns, S. (2017). Health-care-associated infections in neonates, children, and adolescents: an analysis of paediatric data from the European Centre for Disease Prevention and Control pointprevalence survey. The Lancet Infectious Diseases, 17(4), 381-389.

Talaat, M., El-Shokry, M., El-Kholy, J., Ismail, G., Kotb, S., Hafez, S., ... \& Lessa, F. C. (2016). National surveillance of health careassociated infections in Egypt: developing a sustainable program in a resource-limited country. American journal of infection control, 44(11), 12961301.

Abdel-Wahab, F., Ghoneim, M., Khashaba, M., El-Gilany, A. H., \& Abdel-Hady, D. (2013). Nosocomial infection surveillance in an Egyptian neonatal intensive care unit. Journal of Hospital Infection, 83(3), 196-199.

Dhaneria, M., Jain, S., Singh, P., Mathur, A., Lundborg, C., \& Pathak, A. (2018). Incidence and determinants of health careassociated blood stream infection at a neonatal Intensive Care Unit in Ujjain, India: A prospective cohort study. Diseases, 6(1), 14.

Kramer, C., Doellman, D., Blaser, C., Rosenberg, R. H., \& Bernatchez, S. F. (2019). A Quality Improvement Approach in Standardizing Pediatric Central Venous Catheter Dressings and Its Impact on the Reduction of Central Line-Associated Bloodstream Infections and Costs. Journal of the Association for Vascular Access, 24(2), 11-19.
Schmid, S., Geffers, C., Wagenpfeil, G., \& Simon, A. (2018). Preventive bundles to reduce catheter-associated bloodstream infections in neonatal intensive care. GMS hygiene and infection control, 13.

Kulali, F., Çalkavur, Ş., Oruç, Y., Demiray, N., \& Devrim, İ. (2019). Impact of central line bundle for prevention of umbilical catheter-related bloodstream infections in a neonatal intensive care unit: A pre-post intervention study. American journal of infection control, 47(4), 387-390.

Payne, V., Hall, M., Prieto, J., \& Johnson, M. (2017). Care bundles to reduce central line-associated bloodstream infections in the neonatal unit: a systematic review and meta-analysis. Archives of Disease in Childhood-Fetal and Neonatal Edition, fetalneonatal2017.

Ismail, M. E., \& Mostafa, A. I. (2016). KNOWLEDGE AND PRACTICE OF STANDARD PRECAUTIONS AMONG NEONATAL INTENSIVE CARE UNIT HEALTHCARE WORKERS OF MISURATA TEACHING HOSPITAL, LIBYA.

Mohmmed, S. S., \& Yousif, K. I. (2017). Effect of Health Educational Program On Nurses Knowledge \& practice Regarding Infection Control in Neonatal Intensive Care Unit at Pediatric Hospitals in Khartoum State, Sudan 2015.

Batran, A., Ayed, A., Salameh, B., Ayoub, M., \& Fasfous, A. (2018). Are standard precautions for hospital-acquired infection among nurses in public sector satisfactory? Archives of Medicine and Health Sciences, 6(2), 223.

Geberemariyam, B. S., Donka, G. M., \& Wordofa, B. (2018). Assessment of knowledge and practices of healthcare 
Nadia Mohammed Alfar et. al.

workers towards infection prevention and associated factors in healthcare facilities of West Arsi District, Southeast Ethiopia: a facility-based cross-sectional study. Archives of Public Health, 76(1), 69.

Manzo, B. F., Mariano, D. R., Ferreira, F. M. C., Matozinhos, F. P., Simão, D. A. D. S., Costa, A. C. L., \& Corrêa, A. D. R. (2019). Knowledge and behavior of professionals about bundled strategies of central venous catheter. Revista brasileira de enfermagem, 72(1), 50-56.

Pareek, A., Kausik, N. K., Jangir, G., \& Pareek, S. (2018). The Effectiveness of Planned Teaching Program Regarding knowledge on selected Venous access device care among B. Sc Nursing Final year Student: a Quasi-Experimental Study. Asian Journal of Nursing Education and Research, 8(4), 471474.

Dumpa, V., Adler, B., Allen, D., Bowman, D., Gram, A., Ford, P., \& Sannoh, S. (2016). Reduction in Central Line-Associated Bloodstream Infection Rates After Implementations of Infection Control Measures at a Level 3 Neonatal Intensive Care Unit. American Journal of Medical Quality, 31(2), 133-138.

Deshmukh, M., \& Shinde, M. (2014). Impact of structured education on knowledge and practice regarding venous access device care among nurses. Int J Sci Res, 3(1), 895-901.

Freitas, F. T. M., Araujo, A. F. O. L., Melo, M. I. S., \& Romero, G. A. S. (2019). Late-onset sepsis and mortality among neonates in a Brazilian Intensive Care Unit: a cohort study and survival analysis. Epidemiology Infection, 147.

Smulders, C. A., van Gestel, J. P., \& Bos, A. P. (2013). Are central line bundles and ventilator bundles effective in critically ill neonates and children?. Intensive care medicine, 39(8), 1352-1358.

Bannatyne, M., Smith, J., Panda, M., Abdel-Latif, M. E., \& Chaudhari, T. (2018). Retrospective cohort analysis of central line associated blood stream infection following introduction of a central line bundle in a neonatal intensive care unit. International journal of pediatrics, 2018.

McMullan, R., \& Gordon, A. (2016). Impact of a central line infection prevention bundle in newborn infants. infection control \& hospital epidemiology, 37(9), 1029-1036.

Bannatyne, M., Smith, J., Panda, M., Abdel-Latif, M. E., \& Chaudhari, T. (2018). Retrospective cohort analysis of central line associated blood stream infection following introduction of a central line bundle in a neonatal intensive care unit. International journal of pediatrics, 201 